\title{
Spin-Photon Dynamics of Quantum Dots in Two-mode Cavities
}

\author{
Florian Meier* and David D. Awschalon ${ }^{\dagger}$ \\ Center for Spintronics and Quantum Computation, \\ University of California, Santa Barbara, California 93106, USA
}

(Dated: February 21, 2018)

\begin{abstract}
A quantum dot interacting with two resonant cavity modes is described by a two-mode JaynesCummings model. Depending on the quantum dot energy level scheme, the interaction of a singly doped quantum dot with a cavity photon generates entanglement of electron spin and cavity states or allows one to implement a SWAP gate for spin and photon states. An undoped quantum dot in the same structure generates pairs of polarization entangled photons from an initial photon product state. For realistic cavity loss rates, the fidelity of these operations is of order $80 \%$.
\end{abstract}

PACS numbers: 78.67.Hc, 75.75.+a, 42.50.Ct

\section{INTRODUCTION}

The electron spin in quantum dots (QD's) is among the most promising candidates for quantum information processing in the solid state 1.2 Optical selection rules make it possible to control and measure spins in QD's optically ${ }^{3,4,5.6}$ For pairs of QD's embedded in a cavity, in the strong-coupling limit cavity photons can mediate an effective exchange interaction between electron spins $\stackrel{3.4}{=}$ The Faraday rotation of a single photon interacting with an off-resonant QD has recently been discussed for the implementation of Bennet's quantum teleportation scheme and the generation of spin-photon entanglement $\underline{\underline{7}}$ Because the coupling of cavity photons to an offresonant QD is weak, such schemes require long electron spin decoherence times, a high cavity $Q$-factor, and control of the cavity $Q$-factor on a picosecond time-scale.

Recent progress in microcavity design has led to mode volumes close to the theoretical limit $(\lambda / n)^{3}$ and $Q$ factors of order $5 \times 10^{3}$, approaching the strong-coupling

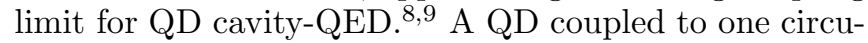
larly polarized cavity mode is described by the JaynesCummings mode ${ }^{10}$ and is expected to show phenomena such as vacuum Rabi oscillations. Here, we theoretically study the coherent dynamics of a QD coupled to two cavity modes 1 and 2 with different spatial distribution and polarization [schematically shown in Fig. I (b) for orthogonal propagation directions]. The design of a cavity with small mode volume and two degenerate, orthogonal modes with circular and linear polarization at the site of the QD is difficult, but possible in principle (see Sec. $\nabla$ below). The aim of this paper is to show that such a system has interesting applications as interface between electron spins and photons because the second cavity mode gives rise to intriguing effects. Most notably, photon transfer between the cavity modes via an intermediate trion state is controlled by the spin state of the QD, opening a wide range of possible applications. We show that (i) for cavity modes in resonance with the heavy hole (hh)-trion transition, entanglement of the electron spin and the cavity modes, i.e., the photon propagation direction is generated. (ii) For cavity modes in resonance with the light hole (lh)-trion tran- (a)

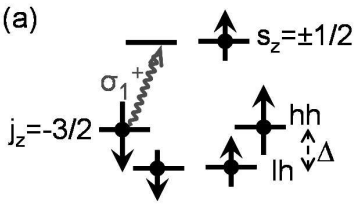

(c)
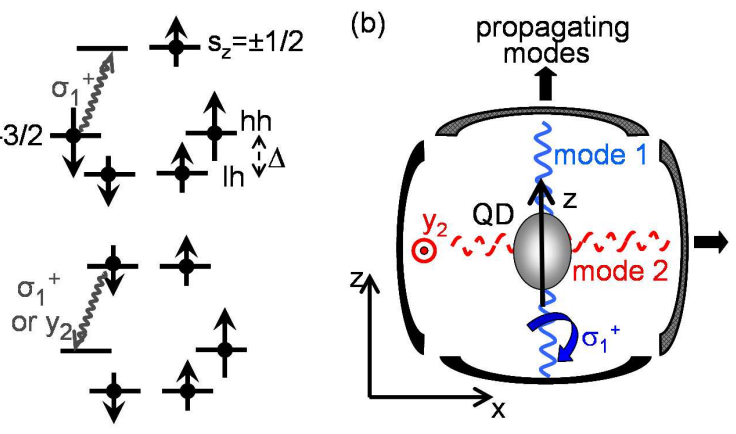

FIG. 1: (a) Characteristic level scheme of, e.g., a CdSe nanocrystal. The crystal anisotropy leads to a splitting $\Delta$ of hh $\left(\left|j_{z}\right|=3 / 2\right)$ and $\mathrm{lh}\left(\left|j_{z}\right|=1 / 2\right)$ states. (b) Schematic representation of the cavity-QD system. The circularly polarized mode $\left|\sigma_{1}^{+}\right\rangle$propagating along direction 1 (aligned with the QD anisotropy axis $z$ ) and the linearly polarized mode $\left|y_{2}\right\rangle$ propagating along 2 are resonant with the hh-trion transition. (c) The trion state can decay by emission of a photon into state $\left|\sigma_{1}^{+}\right\rangle$or $\left|y_{2}\right\rangle$.

sition, the strong-coupling dynamics can be used to implement a SWAP of spin and photon states, an operation which would allow one to transport a spin quantum state over large distances ${ }^{11}$ The quantum state of the photon is encoded in the occupation amplitudes of the two cavity modes. Hence, the system discussed here provides a natural interface between spins and linear-optics quantum information schemes $\stackrel{7.12}{=}$ For cavities with switchable $Q$ factors, the fidelity of all operations, $1-\mathcal{O}(g / \Delta) \simeq 1$, is limited only by off-resonant transitions, where $g$ is the coupling constant for the trion transition and $\Delta$ the hh-lh splitting. However, even for lossy cavities without timedependent control parameters, the fidelity is of order $80 \%$ for realistic cavity loss rates. We also show that (iii) an undoped QD efficiently generates pairs of entangled photons from initial photon product states.

We consider a QD with an anisotropy axis $z$ determined by crystal or shape anisotropy which leads to a splitting $\Delta$ of hh and lh states at the $\Gamma$ point (Fig. 1). The ground state of a singly doped QD is determined by the spin of the excess electron, $\alpha|\uparrow\rangle+\beta|\downarrow\rangle$. In the 
following, we evaluate the dynamics of the QD-cavity system after injection of a photon in state $\left|\sigma_{1}^{+}\right\rangle$at $t=0$. For quantitative estimates, we consider CdSe nanocrystals and adopt the model of Ref. 13 where the anisotropy is treated perturbatively in the quasi-cubic approximation. The coupling constant $g$ for a photon with polarization vector e resonant with the hh (lh)-trion transition is determined by the interband matrix element of the momentum operator, $\mathbf{e} \cdot \hat{\mathbf{p}}$, and the overlap integral of the $1 S_{e}$ and $1 S_{3 / 2}\left(1 S_{1 / 2}\right)$ electron and hh (lh) wave functions. In addition to the strong-coupling criterion that $g / \hbar$ be large compared to the QD spontaneous emission rate and the cavity loss rate, we also assume that $g / \hbar$ is large compared to the hole spin relaxation rate.

In the following, we show that systems such as the one shown in Fig. 1 (b) allow one to generate entanglement between an electron spin and the cavity state (Sec. II), to implement a spin-photon SWAP gate (Sec. IIII), and to efficiently generate pairs of polarization-entangled photons (Sec. IV). In Sec. [V we discuss how a microcavity with the mode structure shown in Fig. I(b) can be engineered and illustrate that the implementation of the schemes discussed in Secs. III III and IV is feasible for microcavities with $Q$-factors exceeding $10^{4}$.

\section{SPIN-PHOTON ENTANGLEMENT}

The interaction of a $\mathrm{QD}$ with a hh valence band ground state [Fig. 1(a)] with the circularly polarized cavity mode propagating along $1,\left|\sigma_{1}^{+}\right\rangle$, and the linearly polarized cavity mode with polarization vector $\mathbf{e}_{y}$ propagating along $2,\left|y_{2}\right\rangle$, is described by a two-mode Jaynes-Cummings model. A photon injected into $\left|\sigma_{1}^{+}\right\rangle$at $t=0$ induces transitions from $|\uparrow\rangle$ to the trion state $\left|X^{-}\right\rangle=\hat{c}_{+}^{\dagger} \hat{c}_{-}^{\dagger} \hat{h}_{-}|G\rangle$, where $|G\rangle$ is the ground state of the QD without excess charge and $\hat{c}_{ \pm}\left(\hat{h}_{ \pm}\right)$the electron annihilation operator for the $1 S_{e}$ conduction band level with $s_{z}= \pm 1 / 2$ (the $1 S_{3 / 2}$ hh level with $j_{z}= \pm 3 / 2$ ). The trion state $\left|X^{-}\right\rangle$has two possible decay paths via emission of a photon in state $\left|\sigma_{1}^{+}\right\rangle$or $\left|y_{2}\right\rangle$ [Fig. प(c)]. In both cases, the QD spin remains unaltered by the cycle of photon absorption and subsequent emission because spin-flip transitions involving the $\mathrm{lh}$-component are dipole forbidden within the model of Ref. 13. The interaction of QD and cavity modes is

$$
\begin{aligned}
\hat{H}_{\mathrm{I}}= & g_{1}\left(\hat{a}_{1} \hat{c}_{-}^{\dagger} \hat{h}_{-}+\text {h.c. }\right) \\
& +g_{2}\left[\hat{a}_{2}\left(\hat{c}_{-}^{\dagger} \hat{h}_{-}+\hat{c}_{+}^{\dagger} \hat{h}_{+}\right)+\text {h.c. }\right],
\end{aligned}
$$

where $\hat{a}_{1}\left(\hat{a}_{2}\right)$ is the photon annihilation operator for mode $\left|\sigma_{1}^{+}\right\rangle\left(\left|y_{2}\right\rangle\right)$ and $g_{1}\left(g_{2}\right)$ the corresponding coupling constant. The free Hamiltonian $\hat{H}_{0}=\delta\left(\hat{a}_{1}^{\dagger} \hat{a}_{1}+\hat{a}_{2}^{\dagger} \hat{a}_{2}\right)$ is determined by the detuning $\delta$ between the photon frequency $\omega$ and the trion transition energy.

While $\left|\downarrow ; \sigma_{1}^{+}\right\rangle$is an energy eigenstate because of Pauli blocking, the QD state $|\uparrow\rangle$ is coupled to both cavity
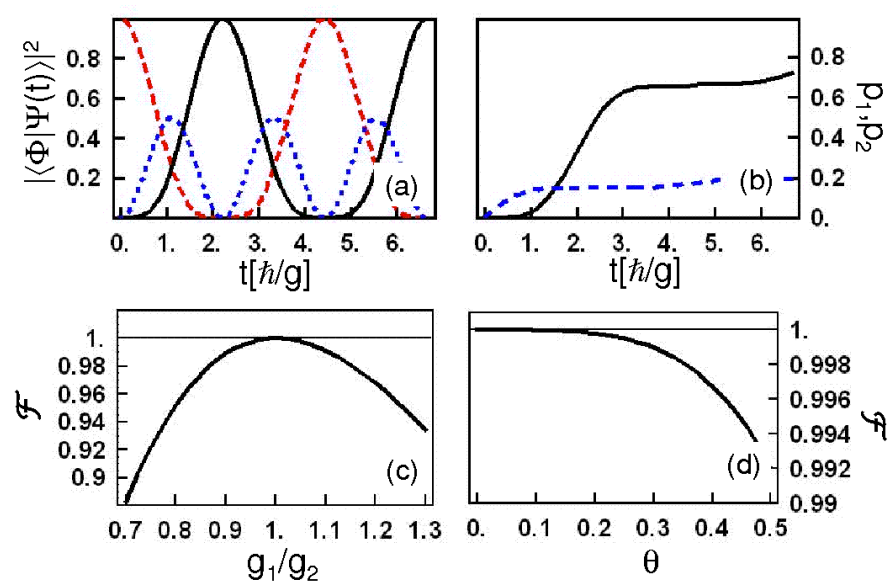

FIG. 2: (a) Time evolution of $|\Psi(0)\rangle=\left|\uparrow ; \sigma_{1}^{+}\right\rangle$. The probabilities $\left|\left\langle\uparrow ; \sigma_{1}^{+} \mid \Psi(t)\right\rangle\right|^{2}$ (dashed), $\left|\left\langle\uparrow ; y_{2} \mid \Psi(t)\right\rangle\right|^{2}$ (solid), and $\left|\left\langle X^{-} ; 0 \mid \Psi(t)\right\rangle\right|^{2}$ (dotted) are shown as a function of time. (b) Probability for photon detection outside the cavity in direction 2 (solid) and 1 (dashed) obtained from numerical integration of Eq. (6) for $\kappa_{1}=0.2 g / \hbar, \kappa_{2}=g / \hbar$, and $\hat{\rho}(0)=\left|\uparrow ; \sigma_{1}^{+}\right\rangle\left\langle\uparrow ; \sigma_{1}^{+}\right|$. (c) Fidelity of spin-photon entanglement generation for $g_{1} \neq g_{2}$. (d) Fidelity of spin-photon entanglement generation as a function of QD misalignment.

modes. The time evolution governed by $\hat{H}=\hat{H}_{0}+\hat{H}_{I}$ leads to transitions from an initial state $\left|\uparrow ; \sigma_{1}^{+}\right\rangle$to $\left|\uparrow ; y_{2}\right\rangle$ via the trion state $\left|X^{-} ; 0\right\rangle$, where $|0\rangle$ is the photon vacuum. Because the dynamics are controlled by the QD spin, photon absorption and re-emission leads to entanglement of the electron spin and the photon cavity mode. This effect is maximal for $g_{1}=g_{2}=g$ and $\delta=0$, where $\frac{14}{4}$

$$
\hat{H}=g\left|X^{-} ; 0\right\rangle\left(\left\langle\uparrow ; \sigma_{1}^{+}\right|+\left\langle\uparrow ; y_{2}\right|\right)+\text { h.c. }
$$

The initial state $|\Psi(0)\rangle=\alpha\left|\uparrow ; \sigma_{1}^{+}\right\rangle+\beta\left|\downarrow ; \sigma_{1}^{+}\right\rangle$evolves to

$$
\begin{aligned}
|\Psi(t)\rangle=\alpha & {\left[\cos ^{2}(E t / 2 \hbar)\left|\uparrow ; \sigma_{1}^{+}\right\rangle-\sin ^{2}(E t / 2 \hbar)\left|\uparrow ; y_{2}\right\rangle\right.} \\
& \left.-(i / \sqrt{2}) \sin (E t / \hbar)\left|X^{-} ; 0\right\rangle\right]+\beta\left|\downarrow ; \sigma_{1}^{+}\right\rangle, \quad(3)
\end{aligned}
$$

where $E=\sqrt{2} g$ [Fig.2(a)]. At times $t_{n}=(2 n+1) h / \sqrt{8} g$, $n$ integer,

$$
\alpha\left|\uparrow ; \sigma_{1}^{+}\right\rangle+\beta\left|\downarrow ; \sigma_{1}^{+}\right\rangle \rightarrow\left|\Psi\left(t_{n}\right)\right\rangle=-\alpha\left|\uparrow ; y_{2}\right\rangle+\beta\left|\downarrow ; \sigma_{1}^{+}\right\rangle .
$$

This demonstrates that, similarly to atom-photon entanglement, ${ }^{15.16 .17}$ spin-photon entangled states of the form of Eq. (4) can be obtained in QD cavity-QED. Alternative schemes for the generation of spin-photon entanglement have been discussed in Refs. 7 and 18 .

According to Eq. (3), the spin-photon entangled state periodically evolves back into the original product state. In order to maintain the state $\left|\Psi_{e}\right\rangle=-\alpha\left|\uparrow ; y_{2}\right\rangle+\beta\left|\downarrow ; \sigma_{1}^{+}\right\rangle$ the photon must be extracted from the cavity. In principle, this is possible by a sudden increase of the cavity loss rate at $t_{n}$. However, cavity loss without time-dependent 
control is also sufficient to generate $\left|\Psi_{e}\right\rangle$ with a fidelity approaching unity if the photon loss rates $\kappa_{1,2}$ for modes $\left|\sigma_{1}^{+}\right\rangle$and $\left|y_{2}\right\rangle$ fulfill

$$
\kappa_{1}<g / \hbar \simeq \kappa_{2}
$$

In this regime, a photon in state $\left|y_{2}\right\rangle$ leaves the cavity before it is scattered back into $\left|\sigma_{1}^{+}\right\rangle$, thus terminating the time evolution in Fig. 2(a) on average after one halfperiod. The condition $\kappa_{1}<g / \hbar$ ensures at least one oscillation be completed. For a quantitative estimate, we integrate the Master equation for the density matrix of the QD-cavity system,

$$
\dot{\hat{\rho}}(t)=-(i / \hbar)[\hat{H}, \hat{\rho}(t)]+\hat{\mathcal{L}}_{l} \hat{\rho},
$$

where cavity loss from $\left|\sigma_{1}^{+}\right\rangle$and $\left|y_{2}\right\rangle$ into free modes propagating along directions 1 and 2 , respectively, is described by the standard Liouville operator

$$
\hat{\mathcal{L}}_{l} \hat{\rho}=-\sum_{i=1,2} \frac{\kappa_{i}}{2}\left(\hat{a}_{i}^{\dagger} \hat{a}_{i} \hat{\rho}+\hat{\rho} \hat{a}_{i}^{\dagger} \hat{a}_{i}-2 \hat{a}_{i}^{\dagger} \hat{\rho} \hat{a}_{i}\right) .
$$

The overall fidelity $\mathcal{F}$ for generation of a spin-photon entangled state as in Eq. (4) is determined by the dynamics of $\hat{\rho}(0)=\left|\uparrow ; \sigma_{1}^{+}\right\rangle\left\langle\uparrow ; \sigma_{1}^{+}\right|$, where photon loss from mode 2 corresponds to successful photon transfer from 1 to 2 . The probability for photon loss along 1 and 2 as a function of time can be obtained from numerical integration of Eq. (6) [shown in Fig. 2(b) for $\kappa_{1}=0.2 g / \hbar$ and $\kappa_{2}=g / \hbar$. For $t \rightarrow \infty$, the probability $p_{2}$ for photon loss into a mode propagating along 2 is calculated from the Fourier-Laplace transform of Eq. (6),

$$
\begin{aligned}
p_{2} & =\kappa_{2} \int_{0}^{\infty} d t\left\langle\uparrow ; y_{2}|\rho(t)| \uparrow ; y_{2}\right\rangle \\
& =\frac{4 \kappa_{2}(g / \hbar)^{2}}{\left(\kappa_{1}+\kappa_{2}\right)\left[4(g / \hbar)^{2}+\kappa_{1} \kappa_{2}\right]} .
\end{aligned}
$$

For the parameters in Fig.2(b), $p_{2}=79 \%$. If the photons propagate freely outside the cavity, the entanglement of the $\mathrm{QD}$ spin and the photon propagation direction is preserved even after photons are ejected from the cavity. In the regime of Eq. (5), the fidelity $\mathcal{F}=p_{2}$ for generating spin-photon entanglement approaches unity.

In the ideal case $p_{2} \simeq 100 \%$, a (maximally entangled) Bell state is obtained for an electron spin prepared in $(|\uparrow\rangle+|\downarrow\rangle) / \sqrt{2}$, which evolves according to $\left(\left|\uparrow ; \sigma_{1}^{+}\right\rangle+\mid \downarrow\right.$ $\left.\left.; \sigma_{1}^{+}\right\rangle\right) / \sqrt{2} \rightarrow\left(-\left|\uparrow ; y_{2}\right\rangle+\left|\downarrow ; \sigma_{1}^{+}\right\rangle\right) / \sqrt{2}{ }^{19}$ We next quantify the entanglement of the final state for a lossy cavity. As long as the photons are not detected outside the cavity and loss in the propagating modes is negligible, the initial state evolves into a pure state, $\stackrel{17.20 .21 .22}{\text { for which the }}$ entanglement $E$ is given by the von Neumann entropy of the reduced density matrix. ${ }^{23,24}$ The entanglement can be expressed in terms of $p_{2}$ in Eq. (8). Defining $\lambda_{ \pm}=$ $\left(1 \pm \sqrt{1-p_{2}}\right) / 2, E\left(p_{2}\right)=-\sum_{\sigma= \pm} \lambda_{\sigma} \log _{2} \lambda_{\sigma}$. Of particular interest are the limiting cases of large and small $p_{2}$, where $\lim _{p_{2} \rightarrow 1^{-}} E\left(p_{2}\right)=1-\left(1-p_{2}\right) / 4 \ln 2+O\left(\left(1-p_{2}\right)^{2}\right)$ and $\lim _{p_{2} \rightarrow 0^{+}} E\left(p_{2}\right)=\left(1+\ln 4-\ln p_{2}\right) p_{2} / 4 \ln 2+O\left(p_{2}^{2}\right)$, respectively. We illustrate the qualitative dependence of $E$ on $\kappa_{2}$ for fixed $\kappa_{1} \ll g / \hbar$. For $g / \hbar<\kappa_{2} \lesssim 4(g / \hbar)^{2} / \kappa_{1}$, loss along direction 2 is dominant for the spin state $|\uparrow\rangle$, such that $p_{2} \simeq 1$ [Fig. 2(b) and Eq. (8)] and $E$ is of order unity. By contrast, for large cavity loss $\kappa_{2} \gtrsim 4(g / \hbar)^{2} / \kappa_{1}$, $p_{2} \simeq 4(g / \hbar)^{2} / \kappa_{1} \kappa_{2}$ approaches zero because the large linewidth of $\left|y_{2}\right\rangle$ renders photon transfer between the cavity modes inefficient. The entanglement $E$ decreases to zero because the photon leaves the cavity along direction 1 irrespective of the spin state on the QD.

Generation of spin-photon entanglement requires fine tuning of the cavity design to ensure $g_{1}=g_{2}$ (Ref. 25) and alignment of the nanocrystal. We next quantify errors for $g_{1} \neq g_{2}$, finite detuning $\delta \neq 0$, QD misalignment, and transitions involving lh states. In the ideal case, an initial state $\left|\uparrow ; \sigma_{1}^{+}\right\rangle$evolves to $\left|\uparrow ; y_{2}\right\rangle$ with $100 \%$ fidelity, while $\mathcal{F}=\max _{t}\left|\left\langle\uparrow ; y_{2}|\exp (-i \hat{H} t / \hbar)| \uparrow ; \sigma_{1}^{+}\right\rangle\right|^{2}$ quantifies the fidelity for non-ideal situations. For $g_{1} \neq g_{2}$, $\mathcal{F}=1-\left[\left(g_{1}^{2}-g_{2}^{2}\right) /\left(g_{1}^{2}+g_{2}^{2}\right)\right]^{2}$, which remains close to unity for $\left|g_{1}-g_{2}\right| /\left|g_{1}+g_{2}\right| \lesssim 1 / 2$ [Fig. 2[(c)]. A finite detuning $\delta$ of the cavity modes relative to the hhtrion transition leads to $\mathcal{F}=1-\mathcal{O}(\delta / g)^{2}$ for $\delta \lesssim g$, which demonstrates the pivotal importance of resonant modes. Misalignment of the QD relative to the photon propagation directions modifies the optical selection rules. For definiteness, consider a nanocrystal with an anisotropy axis rotated by $\theta$ in the plane of the cavity. For $\theta \neq 0$, the coupling energy of $\left|\sigma_{1}^{+}\right\rangle$and transitions from the $j_{z}= \pm 3 / 2$ hh states is $g(1 \pm \cos \theta) / 2$. The dynamics of the system remain periodic for $\theta \neq 0$ and $\mathcal{F}=\left[2(1+\cos \theta) /\left(3+\cos ^{2} \theta\right)\right]^{2} \simeq 1-\theta^{4} / 8$ for $\theta \rightarrow 0$, i.e., the fidelity decreases slowly for $\theta \lesssim 0.5$ [Fig. 2(d)]. Transitions involving lh states are suppressed relative to hh processes by the small factor $g / \Delta$.

\section{SPIN-PHOTON SWAP}

We show next that, for a $\mathrm{QD}$ with a lh valence band maximum, the interaction with two cavity modes allows one to implement a SWAP gate of spin and photon states ${ }^{26}$ We consider a cavity with the geometry shown in Fig. 1(b), for which the circularly polarized mode $\left|\sigma_{1}^{+}\right\rangle$and the linearly polarized mode $\left|z_{2}\right\rangle$ are in resonance with the lh-trion transition while $\left|y_{2}\right\rangle$ is offresonant. While $\left|\uparrow ; \sigma_{1}^{+}\right\rangle$is an energy eigenstate because of Pauli blocking, the state $\left|\downarrow ; \sigma_{1}^{+}\right\rangle$exhibits dynamics similar to Eq. (2). Photon absorption induces transitions to the lh-trion state $\left|X_{l}^{-}\right\rangle=\hat{c}_{+}^{\dagger} \hat{c}_{-}^{\dagger} \widehat{l}_{-}|G\rangle$, where $\widehat{l}_{ \pm}$ annihilates an electron in the lh state with $j_{z}= \pm 1 / 2$ [Fig. [3(a)]. Because both $\left|\sigma_{1}^{+}\right\rangle$and $\left|z_{2}\right\rangle$ are resonant with the trion transition, $\left|X_{l}^{-}\right\rangle$has two different decay channels [Fig. 3(b)]. Optical selection rules imply that, by emission of a photon in state $\left|\sigma_{1}^{+}\right\rangle$, the QD returns to its original spin state $|\downarrow\rangle$ while emission into mode $\left|z_{2}\right\rangle$ leaves the QD in $|\uparrow\rangle$. Hence, transfer of a photon from $\left|\sigma_{1}^{+}\right\rangle$to $\left|z_{2}\right\rangle$ is accompanied by a spin flip on the QD, 

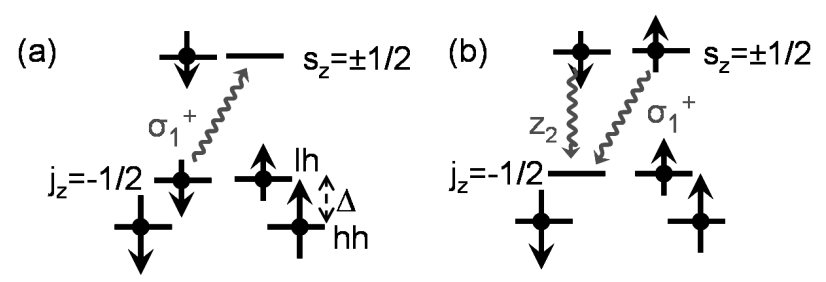

FIG. 3: (a) Absorption process involving lh valence band states. (b) The lh-trion state $\left|X_{l}^{-}\right\rangle$can decay by emission of a photon into mode $\left|\sigma_{1}^{+}\right\rangle$or $\left|z_{2}\right\rangle$. The requirement that $\left|\sigma_{1}^{+}\right\rangle$and $\left|z_{2}\right\rangle$ are the only resonant modes ensures that photon emission into $\left|z_{2}\right\rangle$ is accompanied by a spin flip.

which is described by the Hamiltonian ${ }^{14}$

$$
\hat{H}=g_{1}\left|X_{l}^{-} ; 0\right\rangle\left\langle\downarrow ; \sigma_{1}^{+}\left|-g_{2}\right| X_{l}^{-} ; 0\right\rangle\left\langle\uparrow ; z_{2}\right|+\text { h.c. }
$$

with coupling constants $g_{1,2}$. The dynamics of an initial state $|\Psi\rangle=\alpha\left|\uparrow ; \sigma_{1}^{+}\right\rangle+\beta\left|\downarrow ; \sigma_{1}^{+}\right\rangle$are readily evaluated. In particular, for $g_{1}=g_{2}=g, 27$ we find that at time $t_{n}=h(2 n+1) / \sqrt{8} g$,

$$
\alpha\left|\uparrow ; \sigma_{1}^{+}\right\rangle+\beta\left|\downarrow ; \sigma_{1}^{+}\right\rangle \rightarrow\left|\Psi\left(t_{n}\right)\right\rangle=\alpha\left|\uparrow ; \sigma_{1}^{+}\right\rangle+\beta\left|\uparrow ; z_{2}\right\rangle,
$$

i.e., the QD spin state is swapped onto the photon state encoded in the amplitudes of modes 1 and 2, respectively. This SWAP gate is based on optical selection rules which enforce that photon transfer between the modes is accompanied by a spin flip on the QD. In contrast to schemes such as in Ref. 7, no additional spin measurements are required. The reverse process of Eq. (10), in which the photon state $\alpha\left|\sigma_{1}^{+}\right\rangle+\beta\left|z_{2}\right\rangle$ is transferred onto a QD prepared in an initial state $|\uparrow\rangle$ can also be realized by time evolution under Eq. (9). Then, $\alpha\left|\uparrow ; \sigma_{1}^{+}\right\rangle+\beta\left|\uparrow ; z_{2}\right\rangle \rightarrow \alpha\left|\uparrow ; \sigma_{1}^{+}\right\rangle+\beta\left|\downarrow ; \sigma_{1}^{+}\right\rangle$. Photon states of the form $\alpha\left|\sigma_{1}^{+}\right\rangle+\beta\left|z_{2}\right\rangle$, in which one photon propagates in spatially separated modes, serve as logical basis for linear optics quantum computing $\underline{12}$ The SWAP operation in Eq. (10) provides a natural interface between such coherent photon states and spins. A photon ejected from the cavity can be converted into the standard logical basis $\alpha\left|z_{1}\right\rangle+\beta\left|z_{2}\right\rangle$ by linear optical elements.

Implementation of the spin-photon SWAP gate with unity fidelity requires the interaction between photons and QD be terminated at $t_{n}$. For cavity loss rates which fulfill Eq. (5), no time-dependent control of the cavity parameters is required because cavity loss from mode 2 is sufficient to terminate the dynamics. The fidelity $\mathcal{F}=p_{2}$ derived in Eq. (8) approaches unity. For $\kappa_{1}=0.2 g / \hbar$ and $\kappa_{2}=g / \hbar, \mathcal{F}=79 \%$.

\section{GENERATION OF ENTANGLED PHOTON PAIRS}

Similarly to an atom coupled to two cavity modes, $\frac{28}{,}$ an undoped QD with symmetry axis $z$ at $45^{\circ}$ relative to the propagation directions of modes 1 and 2 [Fig. 4(a)] acts (a)

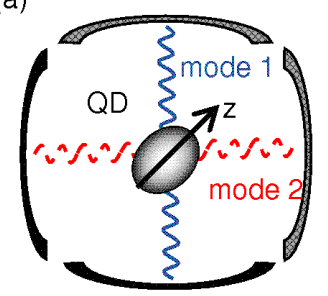

(b)

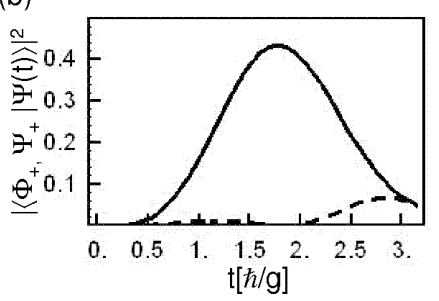

FIG. 4: (a) Setup for the generation of photon-entanglement by strong-coupling dynamics. (b) Projection of $|\Psi(t)\rangle=$ $e^{-i \hat{H} t / \hbar}\left|\sigma_{1}^{+}\right\rangle\left|\sigma_{1}^{-}\right\rangle \otimes|G\rangle$ onto the Bell states $\left|\Psi_{+}\right\rangle$(solid line) and $\left|\Phi_{+}\right\rangle$(dashed line).

as entangler for photon pairs. We consider a QD with the level scheme in Fig. I(a) and assume that the four photon states $\left|\sigma_{1,2}^{ \pm}\right\rangle$are resonant with the lowest (hh) exciton transition. Absorption of photons from mode 1 and re-emission into 2 generates polarization entangled pairs from an initial product state $\left|\sigma_{1}^{+}\right\rangle\left|\sigma_{1}^{-}\right\rangle$. The interaction strength of $\left|\sigma_{1,2}^{+}\right\rangle$and the exciton states $\left|X_{ \pm}\right\rangle=$ $\hat{c}_{\mp}^{\dagger} \hat{h}_{\mp}|G\rangle$ is parameterized by the coupling constants $g(1 \pm 1 / \sqrt{2}) / 2$. Because transitions from $\left|\sigma_{1,2}^{ \pm}\right\rangle$to $\left|X_{ \pm}\right\rangle$ are dominant, for short times $t<h / g$ an initial state prepared by injecting a photon pair $\left|\sigma_{1}^{+}\right\rangle\left|\sigma_{1}^{-}\right\rangle$into the cavity evolves predominantly according to the sequence $\left|\sigma_{1}^{+}\right\rangle\left|\sigma_{1}^{-}\right\rangle \otimes|G\rangle \rightarrow\left(\left|\sigma_{1}^{+}\right\rangle \otimes\left|X_{-}\right\rangle+\left|\sigma_{1}^{-}\right\rangle \otimes\left|X_{+}\right\rangle\right) / \sqrt{2} \rightarrow$ $\left(\left|\sigma_{1}^{+}\right\rangle\left|\sigma_{2}^{-}\right\rangle+\left|\sigma_{1}^{-}\right\rangle\left|\sigma_{2}^{+}\right\rangle\right) \otimes|G\rangle / \sqrt{2}$.

Rigorously, all allowed transitions must be taken into account, $\hat{H}=g \sum_{\alpha, \beta= \pm ; i=1,2} u_{\alpha \cdot \beta} \hat{a}_{i, \alpha}\left|X_{\beta}\right\rangle\langle G|+$ h.c. , where $u_{ \pm}=(1 \pm 1 / \sqrt{2}) / 2$ and $\hat{a}_{i, \pm}$ annihilates a photon in state $\left|\sigma_{i}^{ \pm}\right\rangle$. Because $g$ is small compared to the biexciton shift, biexciton states can be neglected. Integrating the Schrödinger equation, we obtain

$$
\begin{gathered}
|\Psi(t)\rangle=-\frac{1-\cos \left(2 u_{+} g t / \hbar\right) \cos \left(2 u_{-} g t / \hbar\right)}{2 \sqrt{2}}\left|\Psi_{+}\right\rangle \\
-\frac{\sin \left(2 u_{+} g t / \hbar\right) \sin \left(2 u_{-} g t / \hbar\right)}{2 \sqrt{2}}\left|\Phi_{+}\right\rangle+|\tilde{\Psi}\rangle
\end{gathered}
$$

with the Bell states $\left|\Psi_{+}\right\rangle=\left(\left|\sigma_{1}^{+}\right\rangle\left|\sigma_{2}^{-}\right\rangle+\left|\sigma_{1}^{-}\right\rangle\left|\sigma_{2}^{+}\right\rangle\right) \otimes$ $|G\rangle / \sqrt{2}$ and $\left|\Phi_{+}\right\rangle=\left(\left|\sigma_{1}^{+}\right\rangle\left|\sigma_{2}^{+}\right\rangle+\left|\sigma_{1}^{-}\right\rangle\left|\sigma_{2}^{-}\right\rangle\right) \otimes|G\rangle / \sqrt{2}$. $|\tilde{\Psi}\rangle$ represents components with zero photons in one of the modes. Figure 4 shows the projection onto $\left|\Psi_{+}\right\rangle$ (solid line) and $\left|\Phi_{+}\right\rangle$(dashed line) as a function of time. As expected, for $t \lesssim h / g$ the transition to the polarization entangled state $\left|\Psi_{+}\right\rangle$is dominant. At $t_{n}=h n / 4 u_{ \pm} g$, the $\left|\Phi_{+}\right\rangle$component vanishes. While instantaneous reduction of the cavity $Q$-factor at $t_{n}$ would allow one to extract $\left|\Psi_{+}\right\rangle$from the cavity with a fidelity limited only by off-resonant transitions, cavity loss rates $\kappa_{1,2} \simeq g / \hbar$ are also sufficient to terminate the coherent dynamics in Fig. 4(b). Hence, an undoped QD strongly coupled to several modes of a lossy cavity acts as efficient entangler of photon pairs. 


\section{DISCUSSION OF EXPERIMENTAL PARAMETERS}

While our calculations in Secs. III IIII and IVI show that a QD interacting with two cavity modes has interesting applications as interface between spin and photon quantum states, the system is difficult to implement experimentally. Cavities based on Bragg reflectors can sustain degenerate circularly and linearly polarized modes, but mode volumes of order $\lambda^{3}$ are impossible to reach because of diffraction. We show next how the two-mode Jaynes-Cummings Hamiltonian in Eq. (2) [Fig. 1(b)] can in principle be implemented with optical microcavities, where small mode volumes can be achieved. Because the coupling constants in Eq. (2) are determined by the electric fields at the site of the QD only, it is sufficient that the mode $\left|\sigma_{1}^{+}\right\rangle$is circularly polarized locally, at the site of the QD. For definiteness, we focus on the defect modes in a triangular photonic crystal, with a central hole (the defect) with radius $r_{d}$ and dielectric constant $\epsilon_{d}$ which is different from that of all other holes in the triangular lattice. The defect modes with electric field in the cavity plane (TM) and perpendicular to the cavity plane (TE) have been analyzed in detail for some specific realizations of the background and hole medium. ${ }^{29,30,31}$ The defect mode energies are proportional to $r_{d} / \sqrt{\epsilon_{d}}$ and can be tuned across the optical bandgap by varying $r_{d}$ and $\epsilon_{d}$ 29,30,31,32

The following steps allow one to experimentally implement the two-mode Jaynes-Cummings model in Eq. (2): (i) Choose $\epsilon_{d}$ and $r_{d}$ such that a doubly degenerate TE mode (e.g., the $E_{1}$ or $E_{2}$ mode $e^{30}$ ) is degenerate with one TM mode. For a triangular photonic crystal with hexagonal holes, the coexistence of degenerate TE and TM defect modes has recently been demonstrated ${ }^{33} \mathrm{We}$ refer to the modes of the TE-doublet as $\left|\mathrm{TE}_{1 / 2}\right\rangle$ and to the TM mode as $|\mathrm{TM}\rangle$. $\left|\mathrm{TE}_{1}\right\rangle$ and $\left|\mathrm{TE}_{2}\right\rangle$ are related by a $\pi / 2$-rotation ${ }^{30}$ (ii) Identify the set of points $\{P\}=\left\{(x, y) \mid E_{\left|\mathrm{TE}_{1}\right\rangle}=E_{|\mathrm{TM}\rangle}\right\}$ in the cavity plane where the electric field amplitudes $E_{\left|\mathrm{TE}_{1}\right\rangle}$ and $E_{|\mathrm{TM}\rangle}$ of $\left|\mathrm{TE}_{1}\right\rangle$ and $|\mathrm{TM}\rangle$ are equal. The points $\{P\}$ typically form a set of several lines. For every point in $\{P\}$, $\left|\sigma_{1}^{+}\right\rangle=\left(\left|\mathrm{TE}_{1}\right\rangle+i|\mathrm{TM}\rangle\right) / \sqrt{2}$ locally generates an electric field with circular polarization. (iii) In $\{P\}$, identify a point $\left(x_{\mathrm{QD}}, y_{\mathrm{QD}}\right)$ where the electric field amplitude $E_{\left|\mathrm{TE}_{2}\right\rangle}$ of $\left|\mathrm{TE}_{2}\right\rangle$ lies within $30 \%$ of $E_{\left|\mathrm{TE}_{1}\right\rangle} / \sqrt{2}{ }^{34}$ For a $\mathrm{QD}$ at $\left(x_{\mathrm{QD}}, y_{\mathrm{QD}}\right)$ with anisotropy axis oriented perpendicular to the electric field of $|\mathrm{TM}\rangle$ in the cavity plane, the QD-cavity interaction is described by the Hamiltonian Eq. (2) with $\left|g_{2} / g_{1}-1\right| \leq 0.3$, which guarantees a theoretical fidelity of at least $90 \%$ [Fig[2 (c)]. Note that high cavity Q-factors are maintained for a wide range of $\epsilon_{d}$ and $r_{d} 29.30 .31$

Additional requirements for the dynamics discussed in Secs. III and III include a cavity loss rate $\kappa_{2}$ large compared to $\kappa_{1}$ and the injection of a single photon into $\left|\sigma_{1}^{+}\right\rangle$. Because $\left|\mathrm{TE}_{2}\right\rangle$ is predominantly localized along one direction of the photonic crystal, 30 the corresponding cav- ity loss rate $\kappa_{2}$ can be increased by reducing the size of the photonic crystal in this direction, i.e., by removing holes at the outside. While this changes the energies of all three modes, $\left|\mathrm{TE}_{1,2}\right\rangle$ and $|\mathrm{TM}\rangle$, the energy shifts are negligible for cavities with large $Q$-factors. Injection of a single photon into mode $\left|\sigma_{1}^{+}\right\rangle$can be achieved by irradiation of the microcavity with a single-photon source. For TE defect modes in small cubic photonic crystals, the injection efficiency was calculated to be of order $50 \% 35$ Photon injection into $\left|\sigma_{1}^{+}\right\rangle=\left(\left|\mathrm{TE}_{1}\right\rangle+i|\mathrm{TM}\rangle\right) / \sqrt{2}$ is more complicated because coupling efficiencies can be different for TE and TM modes and depend on the direction of incidence relative to the photonic crystal. One can overcome this problem by determining the directions for which the coupling efficiencies for $\left|\mathrm{TE}_{1}\right\rangle$ and $|\mathrm{TM}\rangle$ are comparable, using numerical techniques similar to those in Ref. 35. Alternatively, a source of elliptically polarized photons can be used, where the TE and TM field amplitudes compensate the difference in coupling efficiencies. We also note that a high photon injection efficiency is not required as long as unsuccessful injection attempts can be excluded by post-selection.

For a quantitative estimate, we consider spherical CdSe nanocrystals with a mean radius $a=5 \mathrm{~nm}$. The energy of the lowest exciton state $1 S_{3 / 2}-1 S_{e}$ in an undoped QD is $E_{X}=1.93 \mathrm{eV}$ (Refs. 1336 ) while the trion transition is redshifted by $0.5 \mathrm{meV}$. The hh-lh splitting of a spherical $\mathrm{QD}, \Delta \simeq 20 \mathrm{meV}$, is large compared to the coupling constant $g$. For a mode volume $(\lambda / n)^{3}$, with $n$ the refractive index of the cavity, the electric field amplitude of the cavity modes is of order $E=\sqrt{2 E_{X} n / \epsilon_{0} \lambda^{3}}=$ $5 \times 10^{5} \sqrt{n} \mathrm{~V} / \mathrm{m}$. With the Kane interband matrix element $\left\langle S\left|\hat{p}_{y}\right| Y\right\rangle \stackrel{36}{,} g=(e E / m \omega)\left|\left\langle S\left|\hat{p}_{y}\right| Y\right\rangle\right| \simeq 0.2 \mathrm{meV}$. Strong-coupling phenomena require $g$ to be large compared to both the spontaneous QD emission rate and the cavity loss rates $\kappa_{1,2}$. PL linewidths of $0.12 \mathrm{meV}<g$ have been observed for individual CdSe nanocrystals $\frac{37}{37}$ For cavity $Q$-factors of order $10^{4}, \kappa=\omega / Q \lesssim g / h$. In addition, the phenomena discussed here require a hole spin relaxation time long compared to $h / g \simeq 20$ ps. Recent PL studies of CdSe QD's suggest that hole spin relaxation times are of order $10 \mathrm{~ns} \mathrm{~s}^{38}$ These values show that the strong-coupling dynamics discussed above is within experimental reach for CdSe nanocrystals in a microcavity. The main challenge is to design microcavities with two modes with different polarization, spatial distribution, and loss rates which are strongly coupled to a QD. As shown here, this system would allow one to generate spin-photon entanglement, implement a spin-photon SWAP gate, and create polarization entangled photon states.

\section{Acknowledgments}

We acknowledge helpful discussions with V. Cerletti, R.J. Epstein, S. Ghosh, O. Gywat, Y. Li, and F. Mendoza. This work was supported by ONR and DARPA. 
* Electronic address: meier@physics.ucsb.edu

$\dagger$ Electronic address: awsch@physics.ucsb.edu

1 D. Loss and D. P. DiVincenzo, Phys. Rev. A 57, 120 (1998).

2 S. A. Wolf, D. D. Awschalom, R. A. Buhrman, J. M. Daughton, S. von Molnár, M. L. Roukes, A. Y. Chtchelkanova, and D. M. Treger, Science 294, 1488 (2001).

3 A. Imamoḡlu, D. D. Awschalom, G. Burkard, D. P. DiVincenzo, D. Loss, M. Sherwin, and A. Small, Phys. Rev. Lett. 83, 4204 (1999).

4 A. Imamoḡlu, Fortschr. Phys. 48, 987 (2000).

${ }^{5}$ E. Pazy, E. Biolatti, T. Calarco, I. D'Amico, P. Zanardi, F. Rossi, and P. Zoller, Europhys. Lett. 62, 175 (2003).

${ }^{6}$ P. Chen, C. Piermarocchi, L. J. Sham, D. Gammon, and D. G. Steel, Phys. Rev. B 69, 075320 (2004).

7 M. Leuenberger, M. Flatté, and D. D. Awschalom, condmat/0407499.

8 A. Kiraz, C. Reese, B. Gayral, L. Zhang, W. V. Schoenfeld, B. D. Gerardot, P. M. Petroff, E. L. Hu, and A. Imamoḡlu, J. Opt. B 5, 129 (2003).

9 J. Vučković and Y. Yamamoto, Appl. Phys. Lett. 82, 2374 (2003).

10 R. R. Puri, Mathematical Methods of Quantum Optics (Springer, New York, 2001).

11 J. L. Park, Found. Phys. 1, 23 (1970).

12 E. Knill, R. Laflamme, and G. J. Milburn, Nature 409, 46 (2001).

13 A. L. Efros, Phys. Rev. B 46, 7448 (1992).

14 Terms which are irrelevant for an initial photon state $\left|\sigma_{1}^{+}\right\rangle$ are omitted.

15 J. M. Raimond, M. Brune, and S. Haroche, Rev. Mod. Phys. 73, 565 (2001).

16 B. B. Blinov, D. L. Moehring, L.-M. Duan, and C. Monroe, Nature 428, 153 (2004).

17 B. Sun, M. S. Chapman, and L. You, Phys. Rev. A 69, 042316 (2004).

18 R.-B. Liu, W. Yao, and L. J. Sham, cond-mat/0408148.

19 For simplicity, we denote photons propagating along directions 1 and 2 outside the cavity with the same symbols as the corresponding cavity modes. It is understood that, for $t \rightarrow \infty$, photons always occupy propagating modes outside the cavity.

20 S. J. van Enk, J. I. Cirac, and P. Zoller, Phys. Rev. Lett. 78, 4293 (1997).

${ }^{21}$ W. Lange and H. J. Kimble, Phys. Rev. A 61, 063817
(2000).

${ }^{22}$ Up to two phase factors $\left(\phi_{1}\right.$ and $\left.\phi_{2}\right)$ which are irrelevant for the following quantification of entanglement, the state can be expressed in terms of the loss probability $p_{2}$ derived in Eq. (8): $\left(\left|\uparrow ; \sigma_{1}^{+}\right\rangle+\left|\downarrow ; \sigma_{1}^{+}\right\rangle\right) / \sqrt{2} \rightarrow\left(\sqrt{1-p_{2}} e^{i \phi_{1}}\left|\uparrow ; \sigma_{1}^{+}\right\rangle+\right.$ $\left.\sqrt{p_{2}} e^{i \phi_{2}}\left|\uparrow ; y_{2}\right\rangle+\left|\downarrow ; \sigma_{1}^{+}\right\rangle\right) / \sqrt{2}$.

23 C. H. Bennett, H. J. Bernstein, S. Popescu, and B. Schumacher, Phys. Rev. A 53, 2046 (1996).

${ }^{24}$ W. K. Wootters, Phys. Rev. Lett. 80, 2245 (1998).

${ }^{25} g_{1}=g_{2}$ if $E_{1} / E_{2}=1 / \sqrt{2}$, where $E_{1,2}$ is the electric field amplitude of one photon in $\left|\sigma_{1}^{+}\right\rangle$or $\left|y_{2}\right\rangle$, respectively.

26 The level scheme is determined by the QD shape anisotropy. See Al. L. Efros and A. V. Rodina, Phys. Rev. B 47, 10005 (1993).

${ }^{27} g_{1}=g_{2}$ if $E_{1} / E_{2}=\sqrt{2}$, where $E_{1,2}$ is the electric field amplitude of one photon in $\left|\sigma_{1}^{+}\right\rangle$or $\left|z_{2}\right\rangle$, respectively.

28 C. Wildfeuer and D. H. Schiller, Phys. Rev. A 67, 053801 (2003).

29 V. Kuzmiak and A. A. Maradudin, Phys. Rev. B 57, 15242 (1998).

30 V. Kuzmiak and A. A. Maradudin, Phys. Rev. B 61, 10750 (2000).

31 N. Stojić, J. Glimm, Y. Deng, and J. Haus, Phys. Rev. E 64, 056614 (2001).

32 The detailed dependence of the mode energies on $r_{d}$ and $\epsilon_{d}$ is typically different for TE and TM modes.

33 A. F. Matthews, S. F. Mingaleev, and Y. S. Kivshar, physics/0311018.

34 While no such point is guaranteed to exist, the requirement on the electric field strength of $E_{\left|\mathrm{TE}_{2}\right\rangle}$ is not very stringent. Note also that, in addition to $r_{d}$ and $\epsilon_{d}$, at least two additional parameters can be varied to find such a point, namely the radius of the holes in the triangular photonic crystal and the dielectric constants of the background material.

35 P. R. Villeneuve, S. Fan, and J. D. Joannopoulos, Phys. Rev. B 54, 7837 (1996).

36 A. Ekimov, F. Hache, M. C. Schanne-Klein, D. Ricard, C. Flytzanis, I. A. Kudryavtsev, T. V. Yazeva, A. V. Rodina, and A. L. Efros, J. Opt. Soc. Am. B 10, 100 (1993).

37 S. A. Empedocles, D. J. Norris, and M. G. Bawendi, Phys. Rev. Lett. 77, 3873 (1996).

38 T. Flissikowski, I. A. Akimov, A. Hundt, and F. Henneberger, Phys. Rev. B 68, 161309(R) (2003). 